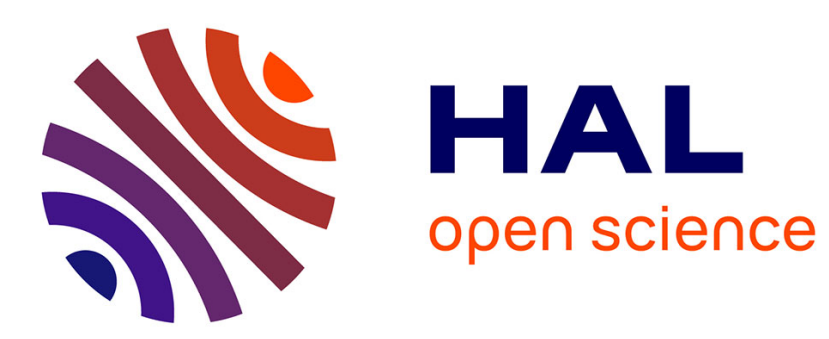

\title{
Elastic and Plastic Aspects of Martensitic Transformations
}

Robert Charles Pond, John Price Hirth

\section{To cite this version:}

Robert Charles Pond, John Price Hirth. Elastic and Plastic Aspects of Martensitic Transformations. Philosophical Magazine, 2010, 90 (07-08), pp.805-819. 10.1080/14786430902956440 . hal-00581013

\section{HAL Id: hal-00581013 https://hal.science/hal-00581013}

Submitted on 30 Mar 2011

HAL is a multi-disciplinary open access archive for the deposit and dissemination of scientific research documents, whether they are published or not. The documents may come from teaching and research institutions in France or abroad, or from public or private research centers.
L'archive ouverte pluridisciplinaire HAL, est destinée au dépôt et à la diffusion de documents scientifiques de niveau recherche, publiés ou non, émanant des établissements d'enseignement et de recherche français ou étrangers, des laboratoires publics ou privés. 


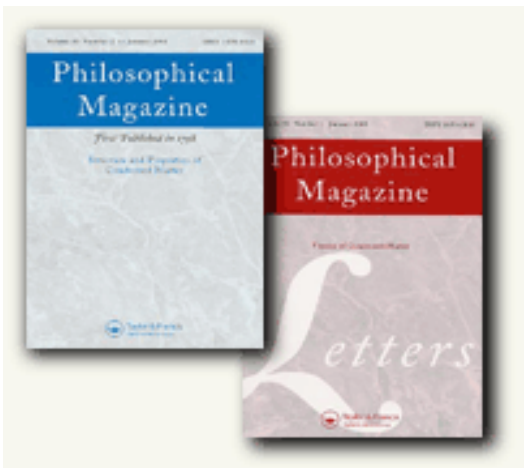

\section{Elastic and Plastic Aspects of Martensitic Transformations}

\begin{tabular}{|c|c|}
\hline Journal: & Philosophical Magazine \& Philosophical Magazine Letters \\
\hline Manuscript ID: & TPHM-09-Feb-0073.R1 \\
\hline Journal Selection: & Philosophical Magazine \\
\hline $\begin{array}{r}\text { Date Submitted by the } \\
\text { Author: }\end{array}$ & 03-Apr-2009 \\
\hline Complete List of Authors: & $\begin{array}{l}\text { Pond, Robert; University of Exeter, School of Engineering, } \\
\text { Computing and Mathematics } \\
\text { Hirth, John; Retired, N/A }\end{array}$ \\
\hline Keywords: & $\begin{array}{l}\text { crystalline interface, deformation, dislocation theory, elasticity, } \\
\text { phase transformations, plasticity }\end{array}$ \\
\hline Keywords (user supplied): & crystalline interface, deformation, dislocation theory \\
\hline
\end{tabular}

\section{今 scholarONE" \\ Manuscript Central}




\title{
Elastic and Plastic Aspects of Martensitic Transformations
}

\author{
R.C. POND* and J.P. HIRTH $\$$ \\ *School of Engineering, Computing and Mathematics, Harrison Building, \\ University of Exeter, North Park Road, Exeter, EX4 4QF, U.K. \\ \$114 E. Ramsey Canyon Rd., Hereford, AZ 85615, USA
}

\begin{abstract}
Elastic and plastic aspects of martensitic transformations are discussed using the topological model. Here the interface comprises an array of transformation dislocations (disconnections) and dislocations (slip/twinning) superposed on coherently strained terraces. Plastic transformation strain arises by virtue of the conservative motion of the defect array, and is quantified directly in terms of the Burgers vectors of the defects. Superposition of the elastic fields of the defects and the coherency strain produces a short-range interfacial distortion field, but only rigid rotation, $\phi$, of the phases at long-range. Furthermore, the treatment shows that, for elastically isotropic phases with similar moduli, elastic relaxations cause the habit plane to differ by $\phi / 2$ from the classical predictions where such relaxations are suppressed. A non-linear analysis is presented suitable for instances of large values of $\phi$. However, the plastic transformation strain, in combination with the relative orientation, $\phi$, corresponds to the classically predicted shape deformation.
\end{abstract}

\section{Introduction}

${ }^{*}$ Corresponding author. Email: r.c.pond@exeter.ac.uk 
The objective of this paper is to discuss elastic and plastic aspects of martensitic transformations where the interface between parent and product crystals is modelled in terms of dislocations. Bullough and Bilby [1] presented the first such treatment in 1956. Their "simple glissile interface" comprises a "surface dislocation" corresponding to a single set of dislocations which move conservatively and thereby cause the necessary lattice-invariant deformation (LID), represented in matrix notation by $\mathbf{P}_{2}$ (for convenience, we use the later nomenclature introduced by Wayman [2]). Motion of the interface also produces the lattice deformation, $\mathbf{B}$, but this is not modelled explicitly in terms of defects. These two homogeneous deformations, along with a rigid body rotation, $\mathbf{R}$, combine into the shape deformation, $\mathbf{P}_{1}$. Under these assumptions, the interface is an undistorted plane, and the shape deformation is an invariant-plane strain (IPS) given by,

$$
\mathbf{P}_{1}=\mathbf{R B} \mathbf{P}_{2} .
$$

They showed furthermore that the total Burgers vector content for such an interface is zero, consistent with the absence of stress in either crystal. These conclusions are in complete accord with the phenomenological theory of martensite crystallography (PTMC), developed contemporaneously by Wechsler, Lieberman and Read [3] and Bowles and MacKenzie [4], which also considers two crystals to be homogeneous and separated by an invariant-plane (IP). In this framework, the total shape change on transformation can be written

$$
\mathbf{P}_{1}=\left(\mathbf{I}+\mathbf{d} \mathbf{p}^{\prime}\right) \text {, }
$$

where $\mathbf{I}$ is the unit matrix, $\mathbf{d}$ is the displacement of all points at unit distance from the interface, and $\mathbf{p}^{\prime}$ is the transpose of the interface normal $[2,3,4]$. Note that, in this view, the interface plane, $\mathbf{p}^{\prime}$, is determined geometrically by the affine rotation and deformations $\mathbf{R}, \mathbf{B}$, and $\mathbf{P}_{2}$. The former two also determine the orientation relationship (OR) between the adjacent crystals.

Since the pioneering works $[1,3,4]$ a large number of microscopical investigations of interface structure have been undertaken (for a review see [5]). Several studies using transmission electron microscopy showed that martensitic interfaces actually comprise two (or more) sets of discrete defects. One set of dislocations (slip or twinning) corresponds to the LID as envisaged above. The second set comprises transformation dislocations [6], or disconnections [7], line defects with both dislocation and step character. A schematic view of such an interface is depicted 
According to the topological model, the elastic distortion field of a static interface can be written as

$$
\Gamma^{s}\left(x^{\prime}, y^{\prime}, z^{\prime}\right)=\left(\begin{array}{lll}
\varepsilon_{x x}^{\prime} & \varepsilon_{x y}^{\prime} & \varepsilon_{x z}^{\prime} \\
\varepsilon_{x y}^{\prime} & \varepsilon_{y y}^{\prime} & \varepsilon_{y z}^{\prime} \\
\varepsilon_{x z}^{\prime} & \varepsilon_{y z}^{\prime} & \varepsilon_{z z}^{\prime}
\end{array}\right)+\left(\begin{array}{ccc}
0 & -\phi_{x y}^{\prime} & \phi_{x z}^{\prime} \\
\phi_{x y}^{\prime} & 0 & -\phi_{y x}^{\prime} \\
-\phi_{x z}^{\prime} & \phi_{y z}^{\prime} & 0
\end{array}\right) .
$$

The strain and rotational distortion components of $\Gamma^{s}\left(x^{\prime}, y^{\prime}, z^{\prime}\right)$ vary from point to point, describing both the short- and long-range fields produced by superposition of the coherency strain and the elastic field of the defect networks. (The $x^{\prime}, y^{\prime}, z^{\prime}$ coordinate frame defines the habit plane, as opposed to the terrace plane frame $x, y, z$ as illustrated in fig.1). At short-range all strains are partitioned between the phases in a manner depending on their relative elastic compliances and the boundary conditions. In the case where the coherency strain is completely accommodated by the network, all component strains except $\varepsilon_{z z}^{\prime}$ vanish at long-range. At short-range, these strains alternate in sign along $x^{\prime}$ and $y^{\prime}$ respectively, in anti-phase across the habit plane, and diminish to zero when $z^{\prime} \approx z_{d}$ is approximately equal to the relevant defect spacing, $d^{D}$ or $d^{L}$ [14]. The magnitude of $\varepsilon_{z z}^{\prime}$ is finite at long-range in the "single 
interface" case, where no constraint by the matrix is present. For $z^{\prime}>z_{d}$ the strain portion of the distortions vanish and the rotational portions are constant and equal to the ancillary tilts, $\phi_{x z}^{\prime}$ and $\phi_{y z}^{\prime}$, and twist, $\phi_{x y}^{\prime}$.

Elastic aspects are discussed in section 2. The method of linear superposition of elastic fields is used in section 2.1, and a non-linear solution, more appropriate for larger strains, is described in section 2.2. Plastic transformation strains are discussed in section 3. A comparison of TM and PTMC predictions is presented and discussed in section 4. The topological character (Burgers vector, $\mathbf{b}$, and step height, h) of disconnections is summarised in Appendix A, along with the reference states employed.

\section{Elastic Aspects}

For clarity, we consider a transformation where no LID needs to be invoked because the two crystals are naturally coherent parallel to $x, x^{\prime}$. The TM is not restricted to such cases; Ma and Pond [15] for example have employed the TM to analyze habit plane variants in Fe alloys that include twist rotation in the habit plane. The TM and PTMC apply to a planar interface between two semi-infinite crystals, a parent $\beta$ phase and a product $\alpha$ phase. The TM method involves selecting a terrace plane of good match. Then candidate disconnections, $\mathbf{b}$ and h, are selected from the coherent dichromatic pattern (see appendix), where the $\beta$ and $\alpha$ crystals are strained to give perfect matching on the terrace plane, with a coherency strain $\varepsilon_{0}=\Delta a / a$ in the y direction. This strain is partitioned to strains $\pm \varepsilon_{0} / 2$ in the two phases. Here $a$ is the atom spacing in the $\mathrm{y}$-direction in the coherent dichromatic complex and $\Delta a$ is the difference between atom spacings in the natural dichromatic complex. The transformation proceeds by the motion of a sequence of such defects, figure 2 . Once the transformation stops, the equilibrium array of disconnections is determined by adjusting their spacing so that there are no long-range elastic stresses or strains for the array. For this aspect, coordinates $\left(x^{\prime}, y^{\prime}, z^{\prime}\right)$, fixed on the habit plane, where the defect spacing is $\mathrm{L}$ as shown, are more convenient. As also depicted in figure 2 , there 
are coherency stresses associated with the terrace segments that can be described as arising from continuous infinitesimal coherency dislocations [16]. No loss in rigor is entailed if one represents the integral of this distribution over $d^{D}$ as a discrete coherency dislocation with Burgers vector $\mathbf{b}_{c y}$ defined using the natural dichromatic pattern (see Appendix A). The "overlap" step h (see appendix) is assumed to be normal to the terrace plane: modifications when this is not the case are discussed in [17].

Figure 2 here.

\subsection{Linear Superposition Solution}

The $b_{\mathrm{z}}$ component of the disconnection has magnitude $\left(h_{\beta}-h_{\alpha}\right)$, so, when this is zero, the Burgers vector of the disconnections in figure 2 is $\mathbf{b}=\left[0, b_{y}, 0\right]$, and that for the coherency dislocations is $\mathbf{b}_{c}=\left[0, b_{c y}, 0\right]$. Hence, $b_{y}=-b_{c y}$ when $d^{D}$ in figure 2 is the equilibrium value that removes misfit. The net strain components are all zero, either in the $(\mathrm{x}, \mathrm{y}, \mathrm{z})$ coordinates or when the strain matrix is transformed to any other set of coordinates, e.g. ( $\left.x^{\prime}, y^{\prime}, z^{\prime}\right)$. The equilibrium condition is that the strains associated with the disconnections, $\pm b_{y} / 2 d^{D}$, are equal and opposite to those of the coherency dislocations $\pm \varepsilon_{0} / 2$. Therefore, the equilibrium condition $b_{y}^{\prime}=-b_{c y}^{\prime}$ is equivalent to $b_{y}=-b_{c y}$, or, alternatively expressed, $\varepsilon_{0}=-b_{y} / d^{D}$, and long-range strains are all zero when this is satisfied. The latter result is the reason why the TM and PTMC agree exactly when $b_{z}=0$. Of course, there would be local dislocation strains within a distance $z^{\prime} \approx d^{D}$ of the interface according with St.Venant's principle.

When $\mathrm{b}_{z}$ is non-zero, $\mathbf{b}=\left[0, b_{y}, b_{z}\right]$, the situation changes. Now both $b_{y}$ and $b_{z}$ contribute to $b_{y}^{\prime}$ and $b_{z}^{\prime}$. When the equilibrium condition $b_{y}^{\prime}=-b_{c y}^{\prime}$ is true, $b_{y} \neq-b_{c y}$ and the equilibrium spacing $d^{D}$ differs from the previous case. The equilibrium condition is now $\varepsilon_{0}^{\prime}=-b_{y}^{\prime} / L$, where the coherency strain in the rotated coordinates is $\varepsilon_{0}^{\prime}$. Also, the $b_{z}^{\prime}$ array, shown in figure 2 , creates a tilt wall with angle $\phi$. If the disconnection array is considered as the superposition of a set of pure steps and a tilt wall, the simple analytical equations of [13] apply. To appreciate this point, we can 
imagine that the actual tilt array $b_{z}^{\prime}$ is cancelled by a hypothetical extrinsic array $b_{e z}^{\prime}$ as shown in figure 3. Since $b_{z}^{\prime} \approx b_{z}$ to linear order, this is equivalent to cancelling $b_{z}$, leaving just the steps h, figure 4 . The basic relation describing the removal of misfit, $\varepsilon_{0}^{\prime}=-b_{y}^{\prime} / L$ (really $\varepsilon_{0}^{\prime} / 2=-b_{y}^{\prime} / 2 L$, but the factors of 2 cancel), then leads to the expression for the equilibrium habit plane angle $\theta$ for this case, given by [13]

$$
-\varepsilon_{0}=\left(b_{y} \tan \theta+b_{z} \tan ^{2} \theta\right) h^{-1}
$$

Figures 3 and 4 here.

The extrinsic dislocations $b_{e z}^{\prime}$ are removed from figure 3 , restoring the tilt wall with the attendant partitioned distortions, which, at long-range, reduce to the partitioned rotations $\pm \phi / 2$. The tilt wall gives a rotation, corresponding to the OR,

$$
\phi=2 \sin ^{-1}\left[\frac{\left(b_{z} \cos \theta-b_{y} \sin \theta\right) \sin \theta}{2 h}-\frac{\varepsilon_{0} \sin \theta \cos \theta}{2}\right] .
$$

In the small $\phi$ limit, this becomes

$$
\phi=\left(b_{z} / h\right) \tan \theta
$$

In the rotated coordinates, the coherency strain is given by $\varepsilon_{0}^{\prime}=\varepsilon_{0}\left(\cos ^{2} \theta-v \sin ^{2} \theta\right) \approx \varepsilon_{0} \cos ^{2} \theta$, where $v$ is Poisson's ratio. The dislocation array has a Poisson term that nearly cancels the coherency term and the ratio $\cos ^{2} \theta / v \sin ^{2} \theta$ is typically of the order $10^{6}$ so the approximation is an excellent one.

Partitioning of elastic distortions is inherent in the above description in several ways. The coherency strain is partitioned to a value $\pm \varepsilon_{0} / 2$ in each crystal. The vector $b_{y}^{\prime}$ is partitioned to a vector $b_{y}^{\prime} / 2$ in each crystal. Finally $b_{z}^{\prime}$ and $\phi$ are partitioned to values $b_{z}^{\prime} / 2$ and $\phi / 2$ in each crystal. In the original treatment, the total 
rotation $\phi$ was assigned to the product phase [13]. However, this was amended in [14] to include the partitioning of the rotation which is necessary as shown in figure 5. Figure 5 here.

Physically, the rotation $\phi$ arises from the summed distortions $\left(\partial \mathrm{u}_{\mathrm{i}} / \partial \mathrm{x}_{\mathrm{j}}\right)$ of the defects. At long range the strain parts of the distortions vanish and only pure rotations are left [17]: these partition equally in the isotropic homogeneous elastic limit. The local strains are present whether the tilt components are $b_{z}^{\prime}$ or $b_{z}$, figure 5. The differences in magnitude of strain, distortion or rotation correspond to a factor $\cos ^{2} \theta$ and vanish to linear order. Partitioning is also nearly the same. Consider the specific case $h_{\alpha}<h_{\beta}$. One can think of the interface separated into two crystals with the free surface step heights $h_{\alpha}$ and $h_{\beta}$, figure 6a. These can also be represented as the superposition of pure steps of height $h=h_{\alpha}$, figure $6 \mathrm{~b}$, and a set of little virtual steps associated with the partitioned dislocations, figure $6 \mathrm{c}$. The superposition of the two arrays shows that, relative to figure $6 \mathrm{~b}$, the superposed tilt causes the habit plane in $\beta$ to increase in this example, while that in $\alpha$ will decrease. The final habit planes, $\omega$, are then given by the superposed results as $\omega=\theta \pm \phi / 2$. For the case where $h_{\alpha}<h_{\beta}$, $\omega_{\alpha}=\theta-\phi / 2$ and $\omega_{\beta}=\theta+\phi / 2$, with $\phi$ positive. The sign of $\phi$ would be negative when $h_{\beta}<h_{\alpha}$.

Figure 6 here.

The results are summarized in figure 7 where habit plane inclinations are plotted as a function of $h_{\beta} / h_{\alpha}$ (the magnitude of $h_{\alpha}$ has been fixed while $h_{\beta}$ is allowed to vary). The PTMC results are also shown in the figure; the habit plane inclination with respect to the $\beta$ phase is $\eta_{\beta}$. For values of $h_{\beta} / h_{\alpha}$ near unity, the habit plane with respect to the $\alpha$ phase (not shown) would be $\eta_{\alpha}=\eta_{\beta}-\phi$. When $h_{\beta} / h_{\alpha}>1, \phi$ is positive and $\eta_{\alpha}$ lies between $\omega_{\alpha}$ and $\omega_{\beta}$. When $h_{\beta} / h_{\alpha}<1, \phi$ is negative and $\eta_{\alpha}$ lies above $\omega_{\alpha}$. We see that the PTMC and TM habit planes differ systematically by $\phi / 2$ for values of $h_{\beta} / h_{\alpha}$ near 1 . When $h_{\beta} / h_{\alpha}$ is larger, the values of $\phi$ differ in the two methods and the habit planes differ by $\approx \phi / 2$. As discussed in Section 4 , the difference of $\phi / 2$ arises because the PTMC, being a continuum analysis, does not include interface structure: the partitioning by $\phi / 2$ arises in the relaxation of the 
interface, figure 5. This difference of $\phi / 2$ is only significant for cases of large $\phi$. However, it is significant in the large $\phi$ case as a first-order correction, unlike the second-order effects discussed next. As a final illustration, the steps in figure $6 \mathrm{a}$ and b can be offset by half the defect spacing without changing associated long-range elastic fields (plastic shear of an equilibrium interface leaves the same elastic equilibrium interface). This is a hypothetical case, which would have higher energy than that of Figure 5, but it again demonstrates the necessity of partitioning as in the TM. The fields of part of the arrays cancel and the excess array has a net Burgers vector $b_{z}{ }^{\prime}$, which partitions to $b_{z}^{\prime} / 2$ as in the TM as described previously. We present these simple physical descriptions to emphasize that the overlap step height is the natural reference state for the superposition problem.

Figure 7 here.

As a specific example, we consider the transformation in "Ti" where the $0.86 \%$ mismatch in the $\mathrm{x}$ direction is neglected. For this example $\mathrm{h}_{\alpha}=0.2556 \mathrm{~nm}$ and $\mathrm{h}_{\beta}=$ $0.2678 \mathrm{~nm}$. The values of $h_{\beta} / h_{\alpha}, \theta, \phi, \omega_{\alpha}, \omega_{\beta}, \eta_{\beta}$ are shown in Table 1. Another example in the table is a hypothetical case with no misfit in the $\mathrm{x}$ direction but where $\mathrm{h}_{\alpha}=0.2556 \mathrm{~nm}$ and $\mathrm{h}_{\beta}=0.2939 \mathrm{~nm}$.

Table 1 here.

\subsection{The Nonlinear Solution}

The superposition solution is accurate to 3 or 4 places in predictions of $\omega$ provided that $|\phi|<\approx 0.8^{\circ}$. This suffices since it is within the experimental scatter in $\omega$ measurements, and of the order of the uncertainties in $\omega$ values in either the TM or the PTMC because of scatter in lattice parameter measurements. The superposition solution has the advantage of being described by the simple analytic equations (4) and (5). In this region the habit planes are those shown between the vertical bars in figure 7 , and vary in a nominally linear manner with respect to the variable $h_{\beta} / h_{\alpha}$. For a greater value of $\phi$, nonlinear effects become important and there is curvature in the lines. For the "Ti" example, the interface is described as $\omega_{\alpha}$ and $\omega_{\beta}$ as shown in Figures 8. A key point is that $\mathrm{L}$ in figure 2 becomes $L_{n}=L / \cos (\phi / 2)$. Also the 
rotation of coordinates to the actual (x', y', z') coordinates is by the angle $\omega=\theta \pm \phi / 2$ instead of $\theta$. For the rotated strains and Burgers vectors in equation (4), a similar modification occurs. The relation $L=h / \sin \theta$ becomes $L_{n}=h_{n} / \sin (\theta \pm \phi / 2)$. Also $\mathrm{h}_{\mathrm{n}} / \mathrm{h}=\tan (\theta \pm \phi / 2) / \tan \theta$, fig. 8a. These all are in the category of geometric nonlinear corrections. Hence, equation (4) becomes, for the $\alpha$ phase,

$$
-\varepsilon_{0}=\frac{\left[b_{y}+b_{z} \tan (\theta-\phi / 2)\right] \tan \theta}{h} .
$$

Equation (2) is similarly replaced by

$$
\phi=2 \sin ^{-1}\left\{\frac{\left[\left(b_{z} \cos (\theta-\phi / 2)-b_{y} \sin (\theta-\phi / 2)\right] \cos (\theta-\phi / 2) \tan \theta\right.}{2 h}-\frac{\varepsilon_{0} \sin (\theta-\phi / 2) \cos (\theta-\phi / 2)}{2}\right\} .
$$

Analogous equations can be used for the $\beta$ phase with $(\theta-\phi / 2)$ replaced by $(\theta+\phi / 2)$.

Evidently, equations (7) and (8) reduce to (4) and (5) in the small $\phi$ limit. Unlike the simple superposition case, equations (7) and (8) must be solved simultaneously by numerical or iterative methods. Explicitly, for $\theta_{i+1}$ and $\phi_{i}$, one can start with the linear result $\phi_{i}=\phi_{1}$ and iterate; a possible procedure is set out in Appendix B. The results for the "Ti" case are listed in Table 2, showing that the simple superposition results are satisfactory for the small $\phi$ case, i.e. for most cases analyzed or measured. Only in exceptional cases such as the $h_{\beta} / h_{\alpha}=1.15$ case, would the nonlinear corrections be needed. An example would be the martensite transformation in $\mathrm{Pu}-\mathrm{Ga}$ alloys, where $\phi \approx 6^{\circ}$ and $h_{\beta} / h_{\alpha}=1.2[18,19]$. In such a case, however, nonlinear elastic corrections would have to be considered as well as the nonlinear geometric corrections. The most obvious effect is that the coherency strains are no longer described by linear elasticity. The strains arise for stresses applied to the free surfaces normal to y to satisfy the free surface boundary condition. The strains are related to these stresses by third and higher order nonlinear elastic constants, an effect that 
becomes important for strains greater than about $0.5 \%$. Anisotropic elastic effects lead to stress tensors that include more components, perhaps all six, for edges and screws, complicating defect interactions, modifying coherency relaxation and producing results analogous to those for isotropic elastic constants at an interface between elastically inhomogeneous crystals. The most important effect in the latter case is to modify partitioning. For example, in the limit that the matrix was infinitely stiff, all among the coherency stresses, the defect stresses and the tilt rotation, if present, would partition to the product phase [20]. Even in the inhomogeneous isotropic elastic case, similar changes in partitioning occur [21]. In both cases [20, 21], distributions of interface line forces may also be needed for the equilibrium description.

Table 2 here.

All of the effects discussed to this point apply to the analysis of the transformation defects only. The same considerations of partitioning of tilt rotations apply in the TM analysis of LID. As mentioned previously, twist rotations and their partitioning are needed when the disconnections or LID include screw components of dislocations [15]. Interactions between disconnections and LID defects, usually neglected, can also lead to complications [22]: line force distributions can again arise in the interface. We mention these possibilities to reinforce the earlier conclusion that the secondorder differences between the PTMC and TM models may not be significant compared to uncertainties arising from approximations in the models.

\section{Plasticity Effects}

In this section we show that the plastic portion of the TM description of the transformation strain agrees with the shape transformation obtained from the PTMC.

For the static equilibrium of Section 2, the elastic strain fields of the disconnections were important in relieving coherency strains. Moving disconnections also serve to produce the plastic transformation strain, converting the parent $\beta$ to a product $\alpha$. The dislocation component of a moving disconnection sweeping an area $\mathbf{A}$ produces an engineering strain per unit volume $\Gamma=\mathbf{b}^{D} \mathbf{A}$. Here, $\mathbf{A}=A \mathbf{n}$ with $\mathbf{n}$ a unit normal to $\mathbf{A}$. Hence a straight disconnection of length $\ell$ moving by $\delta y$ in the $y$ 
direction in the terrace plane coordinates sweeps an area $A=\ell \delta y$, numerically equal to $\delta y$ for a defect of unit length. Motion of a train of disconnections as in figure 2, with $1 / d^{D}$ defects per unit length in the y direction, produces an engineering strain, $\Gamma_{m}^{D}$, given by

$$
\Gamma_{m}^{D}=\left(\begin{array}{ccc}
0 & 0 & 0 \\
0 & 0 & \gamma_{y z} \\
0 & 0 & \varepsilon_{z z}
\end{array}\right)=\frac{\delta y}{d^{D}}\left(\begin{array}{c}
0 \\
b_{y} \\
b_{z}
\end{array}\right)^{D}\left(\begin{array}{lll}
0 & 0 & n_{z}
\end{array}\right)
$$

with $\mathrm{n}_{\mathrm{z}}=1$. Here $\mathrm{b}_{\mathrm{y}}$ and $\mathrm{b}_{\mathrm{z}}$ are the edge components of the disconnection Burgers vector. Uniform motion of unit area of the train by $-\delta y$ transforms a volume numerically equal to $h \delta y / d^{D}$ from $\beta$ to $\alpha$ and translates the habit plane in the $\mathrm{z}^{\prime}$ direction by $\delta z^{\prime}=-\delta y \sin \theta$. In this formalism, an additional, plastic strain $\varepsilon_{y y}$ arises because of the motion along $\mathrm{z}$ of the coherency dislocations with Burgers vector $\mathbf{b}_{c y}$ and line direction $\mathrm{x}$. When the disconnection array moves by $\delta y$, the resulting strain is

$$
\Gamma_{m}^{C}=\left(\begin{array}{ccc}
0 & 0 & 0 \\
0 & \varepsilon_{y y} & 0 \\
0 & 0 & 0
\end{array}\right)=\frac{\delta y}{d^{D}}\left(\begin{array}{c}
0 \\
b_{c y} \\
0
\end{array}\right)\left(\begin{array}{lll}
0 & n_{y} & 0
\end{array}\right) .
$$

Thus, knowing $\mathbf{b}^{D}=\left[0, b_{y}, b_{z}\right]$ and $\mathbf{b}_{c y}$, one can simply write the total engineering deformation at unit distance, produced when the interface sweeps unit volume, as

$$
\mathbf{P}^{T M}=\left(\mathbf{I}+\Gamma_{m}^{D}+\Gamma_{m}^{C}\right)=\left(\begin{array}{ccc}
1 & 0 & 0 \\
0 & 1+\varepsilon_{y y} & \gamma_{y z} \\
0 & 0 & 1+\varepsilon_{z z}
\end{array}\right)
$$

Equation (11) describes the IP deformation transforming the natural $\beta$ crystal into the natural $\alpha$ crystal by disconnection motion along the terrace plane. It corresponds to the transformation matrix $\mathbf{S}$ used in [13] as input to the PTMC calculation; see figure A1(a). 
In the present case, where no LID is invoked, the PTMC shape deformation, equation (1), is simply given by $\mathbf{P}_{1}=\mathbf{R B}$. In other words, the shape deformation expresses the combined effect of the lattice deformation and the OR. The PTMC result is therefore equivalent to the plastic TM transformation deformation combined with rotation of the product by $\phi$. As explained above, in the TM the rigid-body rotation $\phi$ is associated with the elastic distortion fields of the defects at the interface, which is unchanged on average as the train of defects moves. Thus both the rotations connected to the elastic distortions and the associated elastic distortion partitioning relative to the current interface are unchanged by the motion of the habit plane. The PTMC and the TM agree except for the elastic partitioning. That is, the two models agree on the magnitude of the components of the shape change. However the PTMC applies them relative to the interface cut on $\eta_{\beta}$ while the TM applies them relative to $\theta$.

Using expressions (2.7) and (2.8) of reference [14], one can readily confirm that both the "glide" of disconnections across the terraces and the "climb" of the coherency dislocations perpendicular to this are conservative.

\section{Discussion}

The pioneering works of Wechsler, Lieberman and Read [3] and Bowles and MacKenzie [4] are phenomenological and pertain to crystals where elastic displacements are suppressed. Similarly, Bullough and Bilby's surface dislocation model [1] suppresses elastic relaxations, and is hence congruent with the PTMC. By contrast, the TM $[13,14]$ is aetiological, envisioning the interface structure as being partially coherent and transformation to occur by the motion of defects. In this approach plastic and elastic effects are distinguished. Broadly speaking, the plastic transformation strain and OR predicted by the TM for the case considered here is the same as that predicted by the PTMC. Thus, the shape transformation of the PTMC, equation (1), is equivalent to the plastic transformation strain, expression (11), in combination with the OR, expression (4), of the TM. However, consideration of elastic relaxations in the TM describes the partitioned interfacial displacement field and shows that the habit plane consequently differs slightly from the PTMC prediction. One consequence is that the shape transformation according to the TM can no longer be expressed as a simple IPS like expression (2). 
Although the treatment presented here is for a case where no LID is invoked, it can be readily extended to the more general case. The plastic transformation strain produced when slip or twinning dislocations follow the interface can be expressed as an IPS analogous to equation (11). Moreover, their presence affects the OR and habit plane. The combination of IPSs due to disconnection and dislocation motion, along with the overall OR, can be expressed as an ILS, RB. An example of the application of the TM to a transformation involving LID is the case of (575) lath martensite in ferrous alloys [15]. Here, the disconnections and slip dislocations in the interface have predominantly screw character, producing a partitioned twist displacement field near the interface.

The phenomenological theories cannot explain satisfactorily why habit planes sometimes vary with small composition changes of the phases (for example in $\mathrm{Fe}-\mathrm{C}$ alloys) or with the mode of formation (by cold-work or cooling for example) [2, 6]. In these approaches the lattice deformation normally chosen, $\mathbf{B}$, is that corresponding to minimum strains. By contrast, in the TM this is determined primarily by the Burgers vector of the operative disconnections, as encapsulated in expression (10). For a given transformation a multiplicity of disconnections may arise on a viable terrace plane, and hence distinct disconnection/dislocation arrays, leading to distinct habit planes, might arise in differing circumstances. The multiplicity of potential disconnection/dislocation combinations is particularly marked in the case of ferrous alloys [15].

Partitioning of coherency strains has been recognised by many previous workers, e.g. [16]. However, the partitioning of tilt in phase transformations has only been recognised recently [17]. This is an irreversible thermodynamic relaxation in the strain energy of the system. Its incorporation into the TM augments the PTMC, producing an added rotation of the $\beta / \alpha$ habit planes, by $\pm \phi / 2$ when $\phi$ is small. An unrelaxed PTMC interface is depicted in figure 9, but there is no dislocation arrangement that can be superimposed on the TM structure to reproduce it. Extrinsic dislocations could be added to make, say, $\eta_{\alpha}=\theta$ when $h_{\beta} \succ h_{\alpha}$, but then $\eta_{\beta}$ would also equal $\theta$ and not equal the value $\eta_{\beta}=\theta+\varphi$ of figure 7. Similarly in the other cases, one or the other habit plane could be made to agree with figure 9 , but both could not together so agree.

Figure 9 here. 
In many cases the rotation of $\pm \phi / 2$ is a small emendation but in some it is not. We emphasize that these calculated differences apply to the idealized case of a large bicrystal with a planar interface. The partitioning arises from the superposition of surface tractions on the free surfaces normal to $y$ in order to satisfy the free surface boundary condition. This is a good approximation for the case of lath martensite with a large aspect ratio. For lenticular plates, strain partition is resisted by compatibility forces at the plate tips. There the rotations and strains in the parent tend to be suppressed. This would be favored particularly for plates with small aspect ratios. Compatibility strains $\varepsilon_{x x}^{\prime}$ would modify results for both the TM and the PTMC. Rotations would tend to be suppressed in the TM changing the partitioning in the direction to more closely agree with the PTMC. Of course, many other factors enter when plate curvature and compatibility effects become important.

\section{Conclusions}

Elastic and plastic strains are distinguished in the TM treatment of martensitic transformations. Thus the TM extends previous methods (surface dislocations and phenomenological theories) by including elastic relaxation by partition of the tilt wall rotations, $\phi$, when such walls are part of the interface structure. Such relaxations lead to rotations of the habit plane by $\phi / 2$ away from classical predictions. A nonlinear elastic/plastic analysis to determine these rotations is presented, enabling application of the TM to larger values of $\phi$. In the present work a simplified case is considered where no LID is invoked. When LID is involved, partitioning concepts would also apply.

Plastic deformation arises in the TM through motion of defects at the interface. Transformations exclusive of LID effects are considered in detail in this paper. The plastic transformation strain, in combination with the orientation relationship $\phi$, predicted by the TM agrees with the previous methods.

\section{Acknowledgment}

It was a privilege to contribute to the symposium celebrating David Bacon's outstanding career. One of us (RCP) worked with David for over thirty years at the 


\section{Appendix A}

The unit cells of bcc and hcp Ti viewed along $[01 \overline{1}]_{\beta} /[0001]_{\alpha}$ disposed in the Burgers OR are depicted in figure A1(a). These cells exhibit their natural lattice parameters, $a_{\beta}$ and $a_{\alpha}$, respectively, and misfit by $3.8 \%$ along $[111]_{\beta} /[11 \overline{2} 0]_{\alpha}$ and $0.86 \%[01 \overline{1}]_{\beta} /[0001]_{\alpha}$. In the present work, for simplicity, the latter misfit is suppressed. The pattern created when these modified "Ti" lattices interpenetrate is designated the "Natural Dichromatic Pattern", $\beta$ sites being regarded as "white" and $\alpha$ as "black". The Natural DP is used as a reference space in the TM. A second reference space is defined where the $(2 \overline{11})_{\beta} /(1 \overline{1} 00)_{\alpha}$ "terrace" plane with unit normal $\mathbf{n}$ is forced into coherency by a uniaxial strain, $\varepsilon_{y y}$; the result is known as the "Coherent DP'.

The Natural DP is used as the reference state for characterising coherency dislocations, i.e. the notional array of defects producing $\varepsilon_{y y}[14]$. The Coherent DP is used to characterise disconnections at the interface between $\beta$ and $\alpha$, an array of which accommodates $\varepsilon_{y y}[13,14]$. Figure A1(b) is a schematic illustration of such a disconnection. Its Burgers vector is equal to the difference between the translation vectors indicated, $\mathbf{b}=\mathbf{t}(\beta)-\mathbf{t}(\alpha)$, expressed in the $\mathrm{x}, \mathrm{y}, \mathrm{z}$ coordinate frame, and has components along $\mathrm{y}$ and $\mathrm{z}$ in this example. The smaller of the two step heights, $h(\beta / \alpha)=\mathbf{n} \bullet \mathbf{t}(\beta / \alpha), h(\alpha)$ in this case, is designated the "overlap" step height, $\mathrm{h}$. The difference between the step heights defines the $\mathrm{z}$ component of $\mathbf{b}$.

\section{Appendix B}

Because $\tan (\theta-\phi / 2)=\frac{\tan \vartheta-\tan \phi / 2}{1+\tan \vartheta \tan \phi / 2}$, eq. (7) can be written 


$$
\left[b_{y} \tan \left(\phi_{i} / 2\right)+b_{z}\right] \tan ^{2} \theta_{i+1}+\left[\left(h \varepsilon_{0}-b_{z}\right) \tan \left(\phi_{i} / 2\right)+b_{y}\right] \tan \theta_{i+1}+h \varepsilon_{0}=0 .
$$

Inserting $\phi_{i}$ one can solve this expression for the up-dated $\theta_{i+1}$. Then the updated $\phi_{i+1}$ can be found using eq. (8) in the form $\phi_{i+1}=f\left(\theta_{i+1}, \phi_{i}\right)$. Convergence is rapid since $\phi_{i+1}$ depends only weakly on $\phi_{i}$.

\section{References}

[1] R.Bullough and B.A Bilby, Proc. Phys. Soc. 69B 1276 (1956).

[2] C.M.Wayman, Introduction to the Crystallography of Martensitic

Transformations (Macmillian, New York, 1964).

[3] M.S. Wechsler, D.S. Lieberman and T.A. Read, Trans. AIME 197, 1503 (1953).

[4] J.S. Bowles and J.K. Mackenzie, Acta Metall 2, 129 (1954).

[5] J.M.Howe, R.C.Pond and J.P.Hirth, Prog. Mats. Sci., in press.

[6] J.W.Christian, The Theory of Transformations in Metals and Alloys

(Pergammon Press, Oxford, 2002).

[7] J.P.Hirth, J.Phys. Chem. Solids 55 985(1994).

[8] M.G. Hall,H.I. Aaronson and K.R. Kinsman, Surf. Sci.31 257 (1972).

[9] J.M. Howe and D.A. Smith, Acta Metall. Mater. 402343 (1992).

[10] G.B. Olson and M. Cohen, in Dislocations in Solids, edited by F.R.N.

Nabarro, Vol. 7 (North-Holland, Amsterdam, 1986), p. 295.

[11] U. Dahmen, Scripta Metall. 21, 1029 (1987).

[12] I.-W. Chen and Y.-H. Chiao, Acta Metall. 33, 1827 (1985).

[13] R.C. Pond, S. Celotto and J.P. Hirth, Acta Mater. 51, 5358 (2003).

[14] R.C. Pond, X. Ma, Y.W. Chai and J.P. Hirth, in Dislocations in Solids, edited by F.R.N. Nabarro and J.P. Hirth, Vol. 13 (Elsevier, Amsterdam, 2007), p. 225.

[15] X. Ma and R.C. Pond, J. Nucl. Mater. 361, 313 (2007).

[16] G.B.Olson and M.Cohen, Acta Metall. 271907 (1979).

[17] J.P. Hirth, R.C. Pond and J.Lothe, Acta Mater. 54, 4237 (2006).

[18] J.P. Hirth, J.N. Mitchell, D.S. Schwartz and T.E. Mitchell, Acta Mater. 54, 1917 (2006). 
[19] T.G. Zocco, M.F. Stevens, P.H. Adler, R.I.Sheldon and G.B. Olson, Acta Metall. Mater. 38, 2275 (1991).

[20] J.P. Hirth, D.M. Barnett and J. Lothe, Phil. Mag. 40A, 39 (1979).

[21] J. Dundurs, in Mathematical Theory of Dislocations, edited by T. Mura (Am. Soc. Mech. Engin., New York, 1969), p. 70.

[22] J.P. Hirth, R.C. Pond and J. Lothe, Acta Mater. 55, 5428 (2007).

\section{Tables}

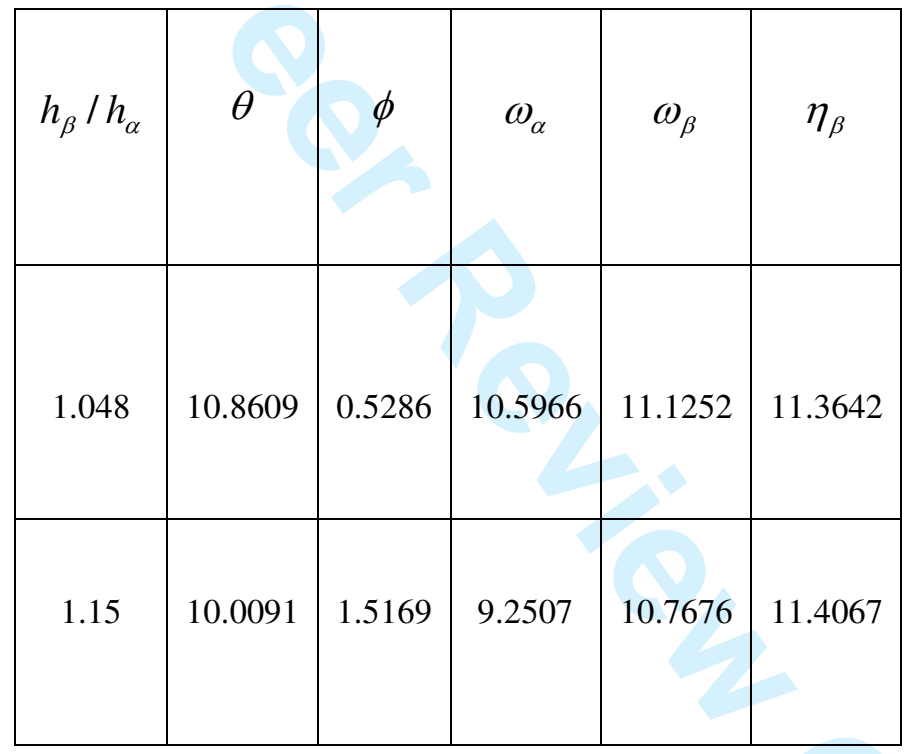

Table 1. Step height ratios and habit plane parameters for "Ti" obtained by the linear superposition method. All angles are expressed in degrees. 


\begin{tabular}{|c|c|c|c|c|}
\hline$h_{\beta} / h_{\alpha}$ & $\theta$ & $\phi$ & $\omega_{\alpha}$ & $\omega_{\beta}$ \\
\hline 1.048 & 10.8727 & 0.5292 & 10.6080 & 11.1373 \\
\hline 1.15 & 10.0934 & 1.5299 & 9.3284 & 10.8583 \\
\hline
\end{tabular}

Table 2. Step height ratios and habit plane parameters for "Ti" obtained by the nonlinear method. All angles are expressed in degrees.

\section{Figure Captions}

Figure 1. Schematic illustration of a parent $(\beta)$-martensite $(\alpha)$ interface showing the terrace segments and defect arrays [13, 14 ]. Coherently strained terraces are reticulated by arrays of disconnections $(\mathbf{b}, \mathrm{h})$ with spacing $\mathrm{d}^{\mathrm{D}}$ and crystal slip dislocations $(\mathbf{b}, 0)$. The terrace and habit (primed) coordinate frames are shown and the line directions of the disconnections, $\xi^{\mathrm{D}}$, and dislocations, $\xi^{\mathrm{L}}$, are parallel to $\mathrm{x}$ and close to y' respectively.

Figure 2. Schematic illustration showing the disconnection content of an interface, with Burgers vector components resolved in the terrace (upper) and habit plane (lower) frames $[13,14]$. The terrace plane is inclined at an angle $\theta$ to the horizontal habit plane. Coherency strain is represented by the equivalent "coherency" defect content, $\mathbf{b}_{c y}$. The $\mathrm{x}$-axis points out of the page.

Figure 3. Schematic illustration of the tilt boundary along the equilibrium interface: the Burgers vectors of the component dislocations are $b_{z}^{\prime}$. A hypothetical array of extrinsic dislocations, $b_{e z}^{\prime}$, exactly cancelling the former tilt is also shown.

Figure 4. Schematic illustration of an array of "overlap" steps defining the habit plane inclination $\theta$. 
Figure 5. Schematic illustration of the partitioning of distortions in both adjacent crystals arising from the dislocation components (a) $b_{z}$, and (b) $b_{z}^{\prime}$. The dashed lines represent bending of planes (elastic distortions) in the vicinity of the dislocations.

Figure 6. Schematic illustration of free-surface step heights, (a) for the separated $\beta$ and $\alpha$ crystals, (b) representation of the common "overlap" step height, and (c) array of virtual steps corresponding to the partitioned tilt wall, $\phi$.

Figure 7. Variation of the TM habit plane angles $\omega_{\alpha}=\theta-\phi / 2$ (dashed line) and $\omega_{\beta}=\theta+\phi / 2$ (dash-dot line) with $h_{\beta} / h_{\alpha}$. The magnitude of $h_{\alpha}$ is fixed while that for $h_{\beta}$ is varied systematically [14]. The PTMC habit plane angle, $\eta_{\beta}$, is also shown (solid line). The linear-superposition treatment is valid in the region between the vertical arrows. In this region $\omega_{\beta}$ and $\eta_{\beta}$ are seen to vary approximately linearly and to differ by $\phi / 2$.

Figure 8. (a) Schematic illustration of the habit plane angle $\omega_{\alpha}=\theta-\phi / 2$ and (b) the habit plane angles $\omega_{\alpha}=\theta-\phi / 2$ and $\omega_{\beta}=\theta+\phi / 2$ after relaxation for the case $h_{\beta} / h_{\alpha}>1$.

Figure 9. Schematic illustration of a habit plane between unrelaxed crystals according to the PTMC.

Figure A1 (a) Illustration of natural bcc and hcp "Ti" lattices exhibiting the Burgers orientation relationship. (b) Schematic illustration of the formation of a disconnection; the $\mathbf{b}$ of the defect with respect to the coherent reference state is the Volterra operation required to create equivalent interfaces on either side of the crystals' surface steps. The 'overlap' step height of the defect is the smaller of the two surface steps, $h(\alpha)$ in this case. The sense vector $\xi$ points out of the page. 


\section{Figures}

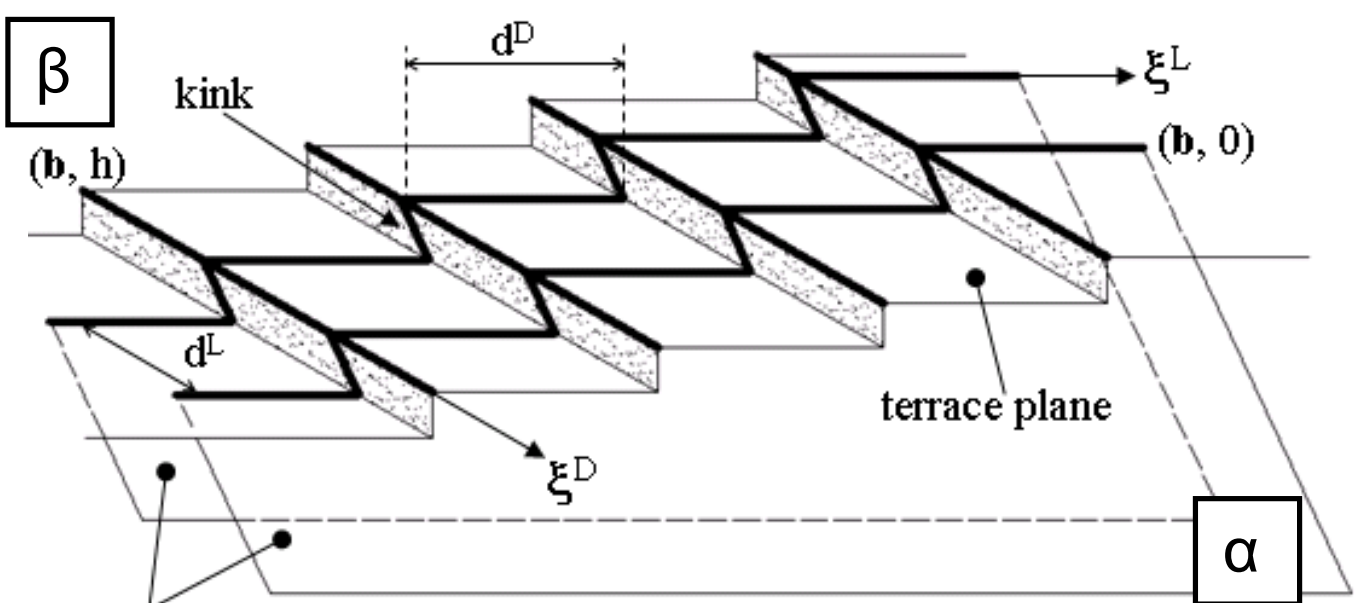

LD glide or twinning planes

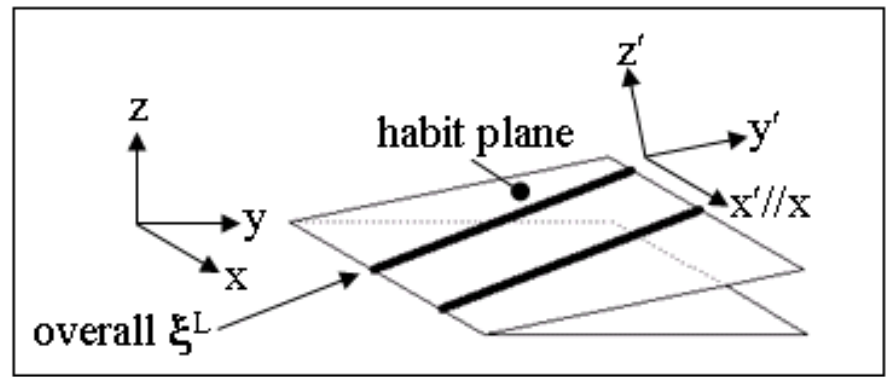

Figure 1

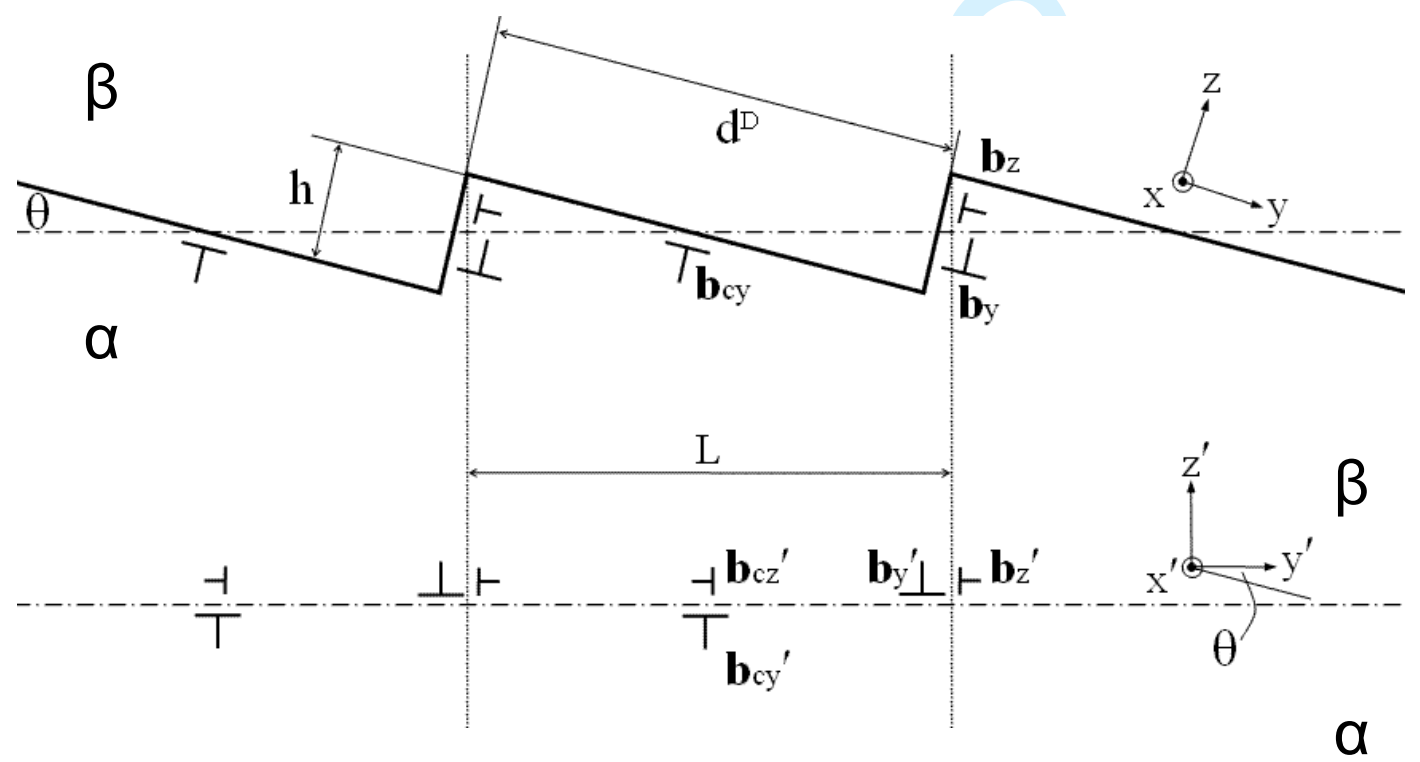


Figure 2

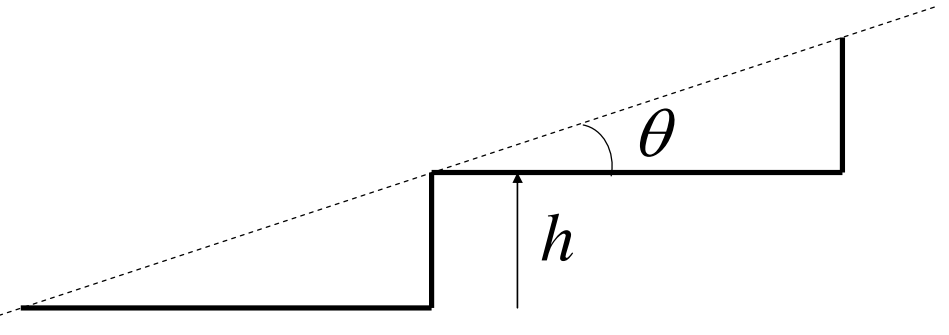

Figure 4 


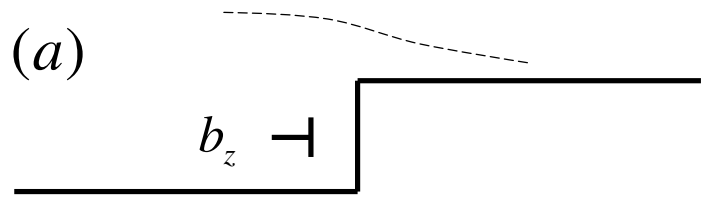

(b)

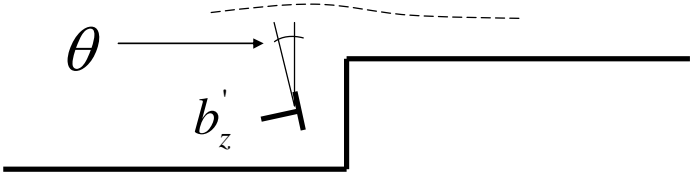

Figure 5
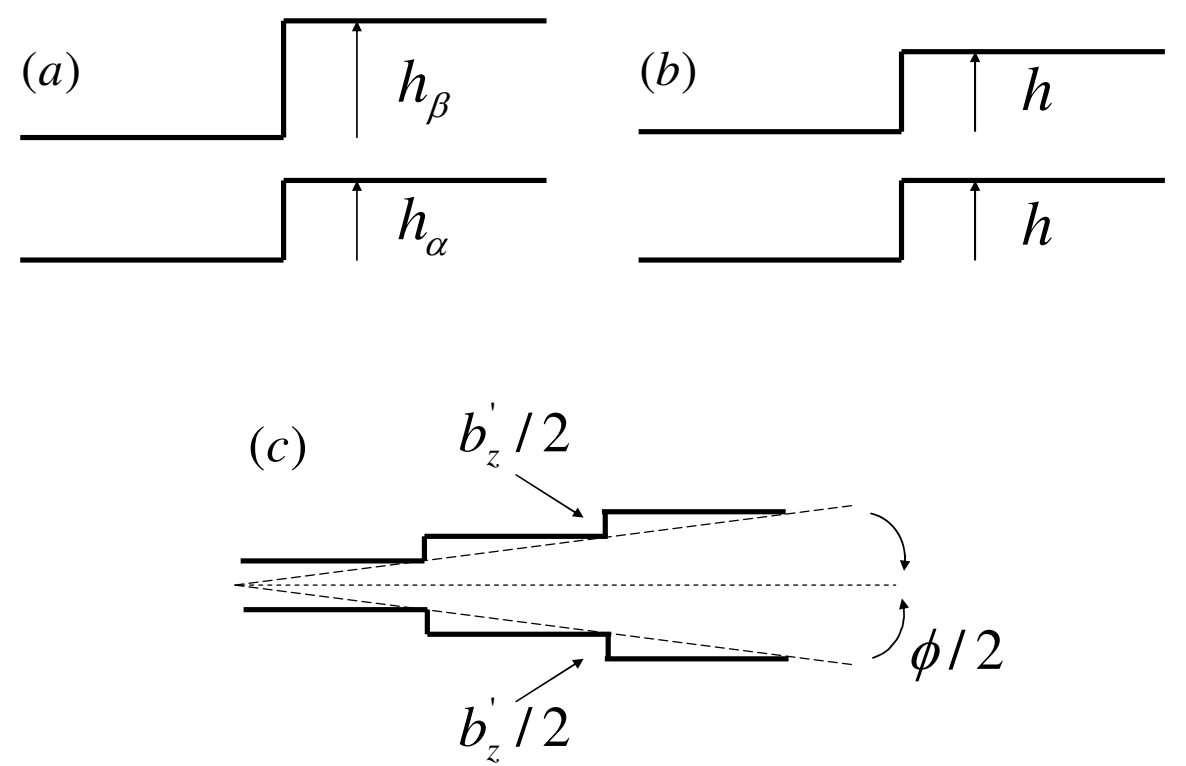

Figure 6 


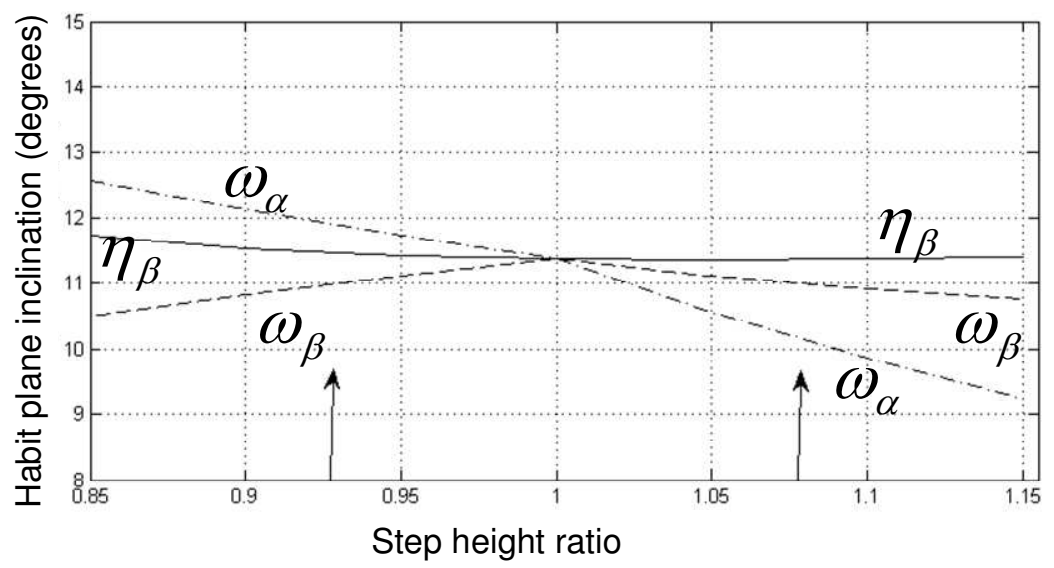

Figure 7 


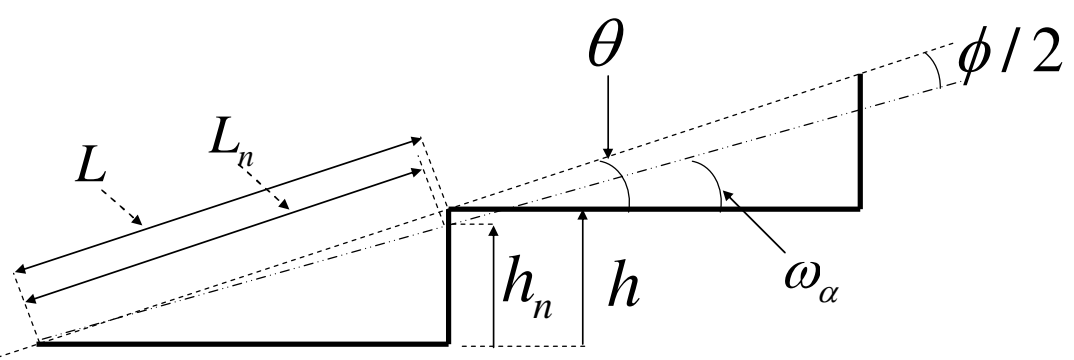

Figure 8(a)

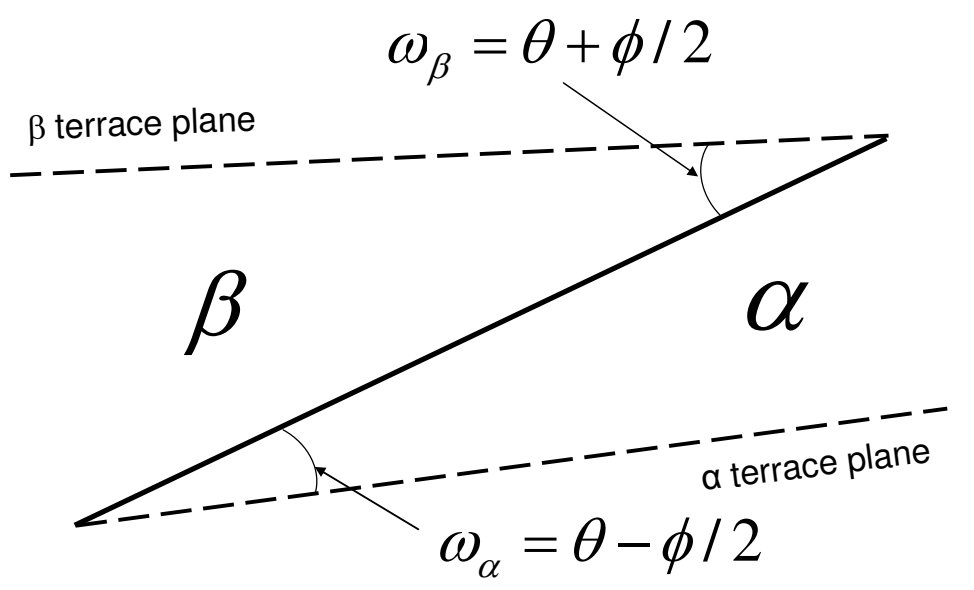

Figure $8(b)$ 


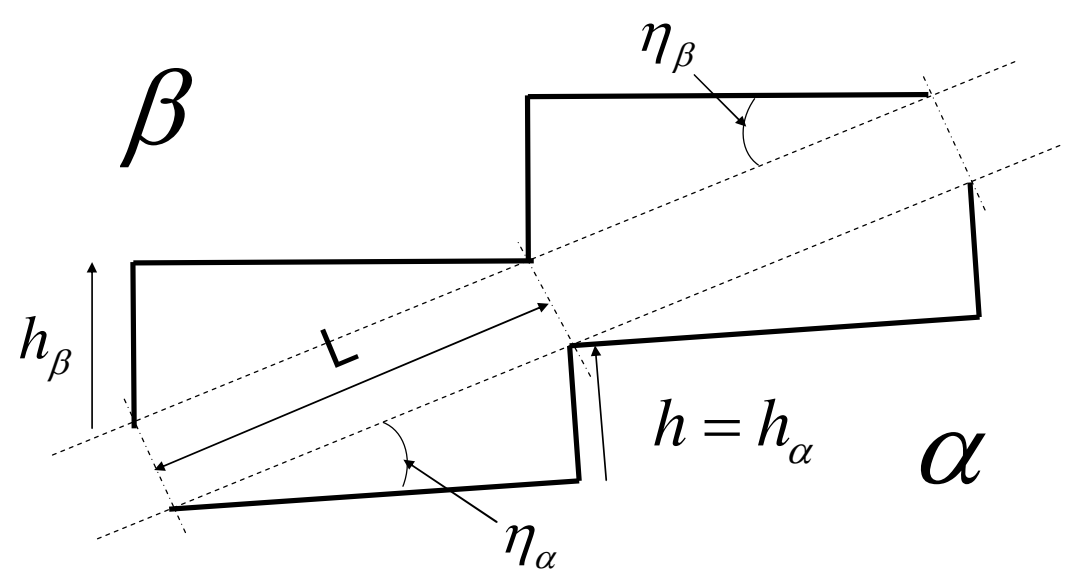

Figure 9

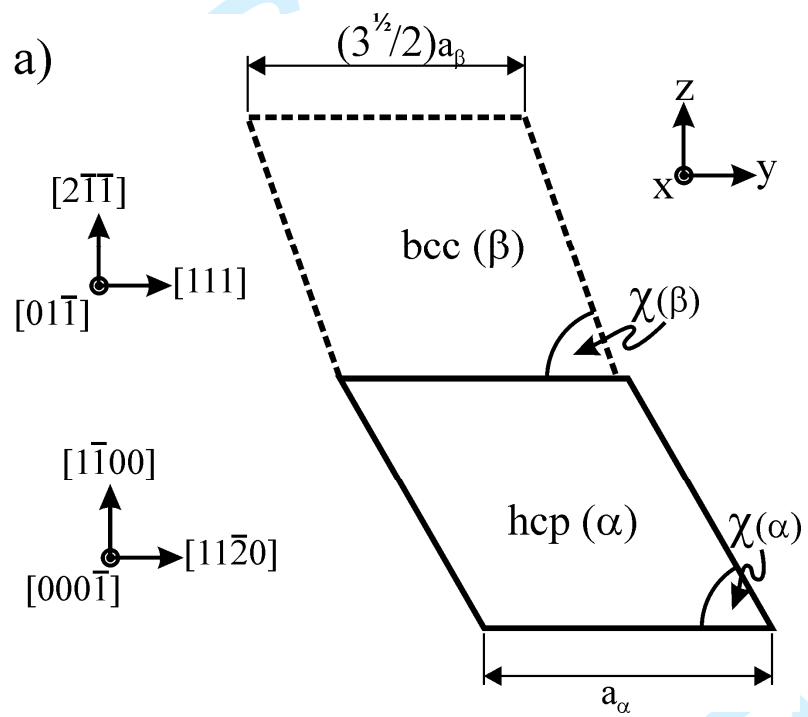

Figure A1(a) 


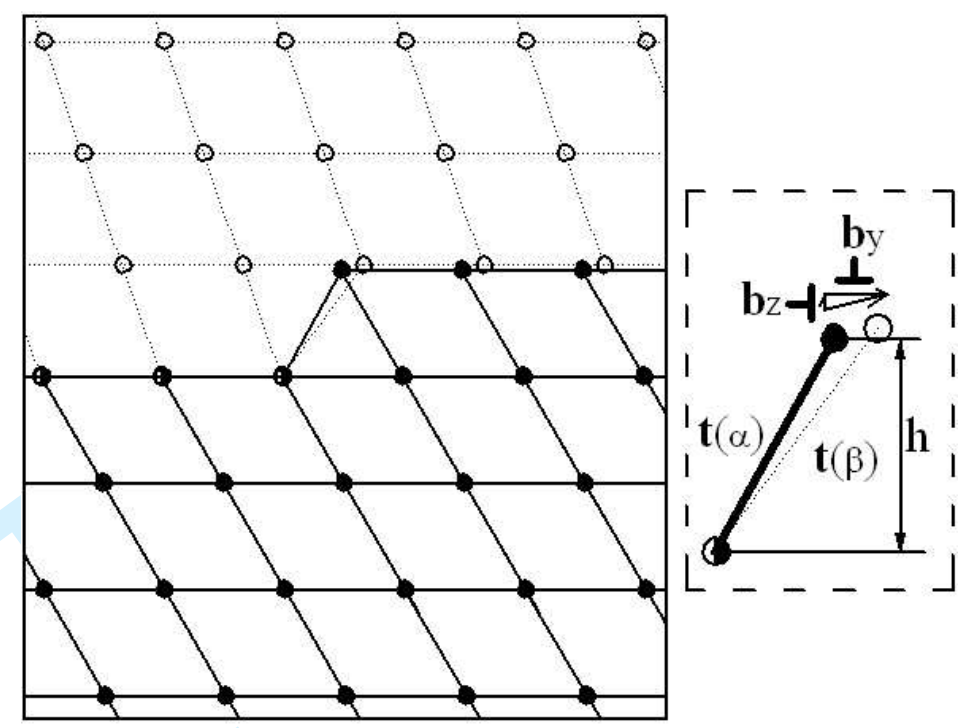

Figure A1(b) 